\title{
Résumé exécutif
}

\section{Minimum wage revisited in the enlarged EU [Le retour du salaire minimum dans l'Europe élargie]}

\author{
Edité par Daniel Vaughan-Whitehead
}

Cet ouvrage livre une analyse originale et approfondie du salaire minimum en Europe du milieu des années 90 à aujourd'hui. Les auteurs expliquent les principales raisons du regain d'intérêt suscité par le salaire minimum et explorent le rôle qu'il pourrait venir jouer dans le nouveau contexte créé par l'élargissement de l'UE, la mondialisation - notamment avec la libéralisation des mouvements de marchandises, de capitaux et de main-d'œuvre - et les changements en matière d'emploi, de rémunération et de relations professionnelles.

Proposant une évaluation des tendances relatives au salaire minimum dans 30 pays européens et réunissant un quinzaine d'études nationales émanant de spécialistes européens reconnus dans ce domaine, ce recueil vient à point nommé pour alimenter le débat actuel: il apporte des preuves tangibles et systématiques de l'évolution du salaire minimum et des changements politiques dans chacun des 15 pays, ainsi que de nouveaux enseignements concernant les effets du salaire minimum, tirés d'études de cas conduites au sein d'entreprises ou secteurs d'activité. Ce développement du salaire minimum avant la crise apporte aussi des enseignements au sein de la crise économique actuelle.

\section{Un regain d'intérêt pour le salaire minimum}

Le salaire minimum est indéniablement revenu parmi les priorités des responsables politiques de l'UE. L'élargissement de l'Union y a certainement concouru. Alors que seuls neuf pays de l'UE des Quinze disposaient d'un salaire minimum légalement établi, l'intégration de 12 nouveaux Etats membres dans l'UE - dont tous, sauf Chypre, disposaient déjà d'un mécanisme de salaire minimum au moment de leur accession - a considérablement augmenté la proportion d'Etats membres dotés d'un salaire minimum légal.

Les vagues successives d'élargissement de l'UE et la concurrence féroce régnant sur les marchés mondiaux ont créé un nouveau contexte suscitant un intérêt accru pour la législation relative au salaire minimum. Le Royaume-Uni et l'Irlande se sont tournés vers un système de salaire minimum, respectivement en 2001 et 2003. L'Autriche a établi aussi un salaire minimum le $1^{\text {er }}$ janvier 2009. La possibilité d'appliquer un salaire minimum national est aussi débattue dans des pays tels que l'Allemagne, l'Italie et la Suède, où les salaires minima sont traditionnellement fixés par négociations collectives sectorielles. 
Les statistiques témoignent de cet intérêt renouvelé pour le salaire minimum. Globalement, au cours de la période 2000-2008, presque tous les pays de l'UE (18 sur 20 ayant un salaire minimum légal) ont augmenté le salaire minimum en termes réels afin d'améliorer le pouvoir d'achat des travailleurs situés au bas de l'échelle salariale. La croissance a été particulièrement impressionnante dans les nouveaux Etats membres, mais également significative dans certains pays de l'UE des Quinze comme la France, la Grèce, l'Irlande, l'Espagne et le Royaume-Uni. Cette politique plus active est confirmée par la hausse du salaire minimum au regard du salaire moyen entre 1995 et 2008 dans 14 des 20 pays bénéficiant d'un salaire minimum légal, avec un ratio moyen pour l'UE des 20 qui augmente de 1,7 pour cent au cours de la période.

\section{Salaire minimum et crise économique}

Alors que nous pouvions craindre que l'intérêt suscité par ce sujet ne s'évapore dans le contexte de la crise économique et financière actuelle, dans les faits, les réactions ont jusqu'ici été très diverses. Dans un certain nombre de pays, la décision a été prise de ne pas ajuster le salaire minimum en 2009, à l'image de l'Irlande, tout comme la Bulgarie, l'Estonie, la Hongrie et la Lituanie, afin de réduire le poids des coûts salariaux pour les employeurs et de les aider par là à maintenir leur activité et à préserver l'emploi. D'autres pays ont décidé de répondre à la crise économique en renforçant le salaire minimum, le considérant comme un moyen de protéger les salaires et le niveau de vie des travailleurs les plus vulnérables, aussi les plus durement frappés par la crise. La Belgique, le Portugal et l'Espagne ont décidé d'augmenter le salaire minimum en 2008 et 2009. De la même manière, la Pologne décidait par accord de dialogue social d'augmenter en 2009 le salaire minimum de près de 15 pour cent afin de stimuler la consommation intérieure.

Ces décisions démontrent ainsi que le regain d'intérêt pour le salaire minimum enregistré dans les pays européens au cours des dix dernières années n'a pas été balayé par la crise. Bien au contraire, cette dernière a conduit nombre de pays à recourir au salaire minimum comme réponse stratégique sur le plan socio-économique, ce qui pourrait contribuer à changer l'utilisation et la configuration de cette institution salariale majeure dans un proche avenir, en Europe, comme peut-être aussi en dehors de l'Europe.

Alors que les dirigeants de la planète réunis au sommet du G20 à Pittsburg les 24 et 25 septembre 2009 ont appelé à «la responsabilité collective pour atténuer l'impact social de la crise», l'OIT dans son pacte mondial pour l'emploi insiste notamment sur le besoin de renforcer les salaires minima afin d'éviter une spirale déflationniste des salaires et de renforcer la protection sociale: «Les gouvernements devraient envisager des options, telles un salaire minimum, qui puissent réduire la pauvreté et les inégalités, accroitre la demande et contribuer à la stabilité économique».

\section{Facteurs d'explication}

Les auteurs identifient quatre facteurs d'explication qui contribuent à redoubler l'intérêt visà-vis des salaires minima.

Le premier facteur est l'accroissement des flux de capitaux et de main-d'œuvre qui découle de l'élargissement de l'UE aux pays d'Europe centrale et orientale, ainsi que de l'intensification 
des flux commerciaux au niveau international. L'arrivée dans l'UE des Quinze de cohortes de travailleurs migrants qui doivent souvent accepter des salaires inférieurs et des conditions de travail plus médiocres que les travailleurs locaux a placé le nouveau réservoir de maind'œuvre ainsi constitué sous les projecteurs.

Deuxième grande source de pression, le recours depuis la fin des années 1990 à de nouvelles formes d'emploi, telles que le travail à temps partiel, le travail en indépendant et l'emploi temporaire. Si cela a stimulé l'emploi dans certains cas, cela a également eu pour effet de confiner certaines catégories de travailleurs dans des conditions de travail et d'emploi précaires et mal rémunérées.

Le déclin progressif du taux de syndicalisation et du champ de la négociation collective que l'on observe dans la plupart des pays de l'UE fournit une troisième explication. Pour contrecarrer leur perte d'influence dans le processus de fixation des salaires - en particulier dans les pays où il n'existe aucun salaire minimum légal - un certain nombre de syndicats ont poussé à l'introduction de cet outil réglementaire afin de mieux influencer les salaires réels et le pouvoir d'achat des travailleurs.

Les tendances salariales offrent une quatrième explication du regain d'intérêt pour le salaire minimum. Dans nombre de pays européens, la modération salariale, la baisse de la part des salaires dans le PNB, l'augmentation des inégalités salariales et un nombre croissant de travailleurs à bas salaires ont soudainement attiré l'attention sur la nécessité de donner un coup de pouce à l'évolution des salaires. En particulier, le phénomène des travailleurs pauvres a contribué à placer la question du salaire minimum sur l'agenda politique d'un certain nombre de pays. Les études nationales présentées dans cet ouvrage identifient les effets réels de la fixation d'un salaire minimum, non seulement sur les bas salaires mais aussi sur l'emploi, les disparités salariales, la négociation collective et les migrations.

Le salaire minimum se révèle être un outil approprié pour gérer à la fois les mouvements d'émigration et d'immigration, comme le montrent les études de cas de l'Irlande et de la Pologne: alors que cela aide le pays d'accueil à attirer les travailleurs migrants, en particulier dans les secteurs où la main-d'œuvre fait défaut, une hausse progressive du salaire minimum dans le pays d'origine peut venir limiter l'expatriation de la main-d'œuvre. En protégeant à la fois les migrants et les travailleurs locaux de rémunérations trop faibles, le salaire minimum représente aussi un rempart contre le dumping social, une dimension qui pourrait encore gagner en pertinence avec la libéralisation des services au sein de l'UE élargie. Enfin, dans certaines conditions, le salaire minimum national pourrait contribuer à renforcer plutôt qu'à supplanter la négociation collective.

\section{Vers un salaire minimum unique dans I'UE?}

Les auteurs abordent aussi la question de savoir s'il devrait y avoir une harmonisation entre les Etats membres, voire un salaire minimum commun à l'UE. C'est une possibilité qui a été mise en avant par certains dirigeants de l'UE et débattue au Parlement européen. Ces initiatives à l'échelon européen sont également motivées par la place qu'occupe le salaire minimum dans l'UE élargie, où plus de 5 millions de travailleurs sont déjà couverts par un salaire minimum légal. 
Cependant, la plupart des observateurs s'accordent sur le fait qu'un salaire minimum unique n'est pas souhaitable au niveau de l'UE, du moins pas aujourd'hui, étant donné les différences qui prévalent entre les montants des salaires minima selon les pays. Dans le même temps, des propositions ont vu le jour en faveur de l'introduction généralisée d'un salaire minimum national et de l'élaboration d'un certain nombre de principes de base communs dans ce domaine, sur par exemple des aspects aussi fondamentaux que l'étendue et les bénéficiaires du salaire minimum (certaines catégories de travailleurs continuant à être exclues du salaire minimum national dans plusieurs pays), le lien qui pourrait être recommandé entre le salaire minimum et des indicateurs économiques et sociaux, tels que le salaire moyen, ou le PNB par habitant, la proportion des travailleurs à bas salaires ou le seuil de pauvreté.

En dressant un bilan comparatif des méthodes de fixation des salaires dans les pays de l'UE, ce livre permet aussi de mieux cerner les conditions optimales, les bonnes pratiques et les leçons politiques dans ce domaine, un exercice particulièrement utile afin de mieux répondre aux demandes croissantes d'assistance en matière de détermination du salaire minimum.

Copyright (C) Organisation internationale du Travail

Ce résumé ne constitue pas un document officiel de l'Organisation internationale du Travail. Les opinions exprimées ne reflètent pas nécessairement les vues de l'OIT. Le désignations utilisées n'impliquent de la part de l'OIT aucune prise de position quant au statut juridique de tel ou tel pays, zone ou territoire, ou de ses autorités, ni quant au tracé de ses frontières. La mention ou la non-mention de telle ou telle entreprise ou de tel ou tel produit ou procédé commercial n'implique de la part de l'OIT aucune appréciation favorable ou défavorable.

Le texte peut être librement reproduit, à condition d'en mentionner la source.

Département de la communication et de l'information publique

Bureau international du Travail

4 route des Morillons, 1211 Genève 22, Suisse

Pour plus d'information, visitez notre site web www.ilo.org 